\title{
Jets in $\mathrm{Pb}-\mathrm{Pb}$ collisions at ALICE
}

\author{
Oliver Busch ${ }^{1,2, a}$ on behalf of the ALICE collaboration \\ ${ }^{1}$ University of Tsukuba, Tsukuba, Japan \\ ${ }^{2}$ Physikalisches Institut, Ruprecht-Karls-Universität Heidelberg, Heidelberg, Germany
}

\begin{abstract}
We report results on jet production in $\mathrm{Pb}-\mathrm{Pb}$ collisions at $\sqrt{s_{N N}}=2.76 \mathrm{TeV}$ from the ALICE collaboration. The nuclear modification factor of jets reconstructed with a resolution parameter $\mathrm{R}=0.2$ shows a strong, centrality dependent suppression with respect to pp collisions. The azimuthal anisotropy of charged jet production relative to the event plane is studied. In semi-peripheral events, a significant $v_{2}^{\text {ch jet }}$ is observed, indicating path-length dependent in-medium parton energy loss. The measurement of jet shapes allows characterizing the intra-jet momentum flow of quenched jets. The measured radial moment and dispersion of the constituent transverse momentum distribution indicate a collimation and hardening of the jet core in $\mathrm{Pb}-\mathrm{Pb}$ relative to $\mathrm{pp}$ collisions.
\end{abstract}

\section{Introduction}

Jets are collimated sprays of particles associated with hard-scattered partons. The study of jet production and fragmentation allows us to test our understanding of perturbative and non-perturbative aspects of QCD. In heavy-ion collisions, jets produced in the initial stage probe the early hot and dense quark-gluon plasma phase of the fireball evolution. Interactions with the medium result in collisional energy loss and give rise to additional induced radiation, which emitted at small angles inside the jet cone can lead to a broadening of the jet profile. Jet reconstruction aims to capture the full dynamics of jet quenching and to quantify the in-medium jet energy loss. Measurements of the jet shape characterize the intra-jet momentum flow, and help to distinguish between a possible jet collimation or broadening as a consequence of jet quenching.

The path-length dependence of parton energy loss is sensitive to the underlying energy-loss mechanism. For collisional energy loss, the amount of lost energy depends linearly on the path-length, while for radiative energy loss [1], the dependence is quadratic due to interference effects. Some strong-coupling models based on the AdS/CFT correspondence [2,3] suggest an even stronger pathlength dependence. Experimentally, the dependence can be studied by measuring jet production relative to the orientation of the event plane, defined via the symmetry axis of the initial nucleon distribution of the collision overlap region.

\section{Jet Reconstruction in ALICE}

Charged jets are reconstructed in the ALICE central barrel from primary charged particle tracks measured in the Inner Tracking System (ITS) and the Time Projection Chamber (TPC). Tracks with trans-

\footnotetext{
ae-mail: o.busch@gsi.de
} 
verse momentum $p_{\mathrm{T}}>150 \mathrm{MeV} / c$ in the pseudo-rapidity interval $|\eta|<0.9$ are clustered with the FastJet [4] anti- $k_{\mathrm{T}}$ algorithm using a boost invariant $p_{\mathrm{T}}$ recombination scheme. We use different values of the jet resolution parameter from $R=0.2$ to $R=0.4$. Jets are selected so that they are fully contained within the detector acceptance. For full jet reconstruction, we include neutral particles (mostly neutral pions) reconstructed with the ALICE Electromagnetic Calorimeter (EMCal). Clusters in the EMCal acceptance $|\eta|<0.7$ and $1.4<\varphi<\pi$ with a transverse energy $E_{\mathrm{T}}>300 \mathrm{MeV}$ are used. The energy of charged particles pointing to EMCal clusters is subtracted to avoid double-counting. The jet shape observables are measured with charged particles.

Jet reconstruction in heavy-ion collisions proceeds against a large background from the underlying event uncorrelated to hard parton scattering. The average charged background energy density is evaluated event-by-event from the median $p_{\mathrm{T}}$ density of FastJet [4] $k_{\mathrm{T}}$ clusters and subtracted jet-byjet. For charged+EMCal jet reconstruction, the charged background density is scaled to the level of charged+neutral particles.

The (charged) jet spectra, and shape distributions, are corrected for detector effects to the (charged) primary particle level, including the missing energy from neutrons and $\mathrm{K}_{L}^{0}$ in the measurements that include the EMCal. The impact of the contribution of background fluctuations to the jet energy resolution is corrected along with the detector effects by unfolding. Background fluctuations also give rise to low $p_{\mathrm{T}}$ 'combinatorial' jets composed of background particles, unrelated to hard parton scattering. Their contribution can be suppressed, e.g. by introducing a fragmentation bias requiring a high transverse momentum jet constituent or by choosing a small jet radius to minimize the background contribution.

\section{Jet Nuclear Modification Factor}

In Fig. 1 [5], the charged+EMCal jet nuclear modification factor $R_{\mathrm{AA}}$ is shown, defined as the ratio of the measured jet spectra in $\mathrm{Pb}-\mathrm{Pb}$ collisions to the pp reference cross section scaled by the nuclear thickness function which represents the number of binary collisions [6]: $R_{\mathrm{AA}}\left(p_{\mathrm{T}}\right)=\frac{1}{\left\langle T_{\mathrm{AA}}\right\rangle} \frac{\mathrm{d}^{2} N_{\text {jet }} / \mathrm{d} p_{\text {T.jet }} \mathrm{d} \eta}{\mathrm{d}^{2} \sigma_{\text {jet }}^{\mathrm{Pp}} / \mathrm{d} p_{\mathrm{T}, \mathrm{jet}} \mathrm{d} \eta}$. In this measurement, jets with $R=0.2$ are used, and a minimum transverse momentum, $p_{\mathrm{T}}>5 \mathrm{GeV} / c$, is required for the leading charged constituent. The systematic uncertainties are divided into correlated uncertainties and shape uncertainties. The correlated uncertainties result dominantly from uncertainties on the jet energy scale, such as the uncertainty of the tracking efficiency, whereas the shape uncertainties are related to the unfolding and can distort the slope of the spectrum.

The measured $R_{A A}$ is significantly smaller than unity, indicating a strong suppression of jets in $\mathrm{Pb}-\mathrm{Pb}$ collisions. The observed value of the jet nuclear modification factor is similar to the $R_{A A}$ of charged hadrons [15], which indicates that a significant fraction of the lost parton energy is not recovered inside the jet cone. A comparison of the $R_{\mathrm{AA}}$ for the $10 \%$ most central events (left panel) and semi-peripheral events (right panel) allows studying the centrality dependence of the suppression. For more central collisions, a smaller $R_{\mathrm{AA}}$ is observed, indicating stronger suppression.

We also show a comparison of the results to calculations from different models. The first model, YaJEM [8], uses a 2+1D hydrodynamical model to calculate medium transport coefficients which describe the coupling between partons and medium and modify the kinematics of the virtual partons in the evolving parton shower through an increase in virtuality. The second model, JEWEL [9], models energy loss in the presence of a QCD medium microscopically through multiple scattering and gluon radiation, including a Monte Carlo (MC) implementation of LPM interference effects. Despite their different approaches, both calculations are found to reproduce the jet suppression. YaJEM, however, exhibits a slightly steeper increase with jet $p_{\mathrm{T}}$ than the data. 


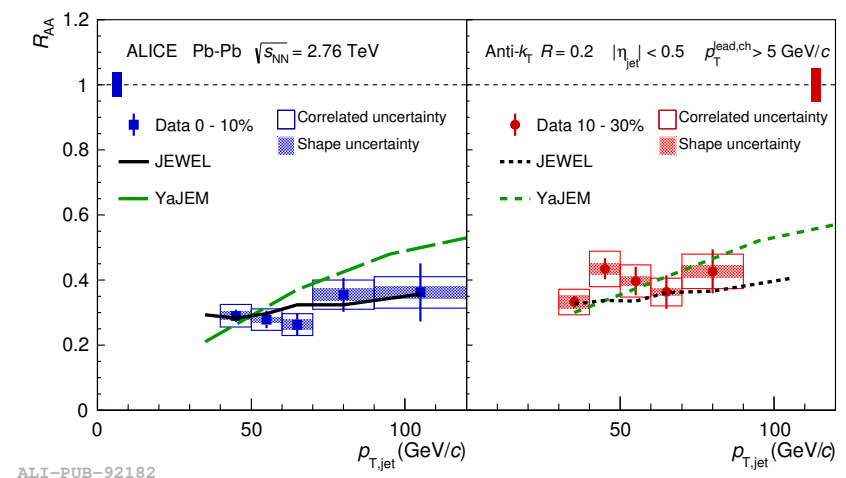

Figure 1. Nuclear modification factor $R_{A A}$ for $R=0.2$ jets [5] with a leading track requirement of $5 \mathrm{GeV} / \mathrm{c}$ in $0-10 \%$ (left) and $10-30 \%$ (right) most central $\mathrm{Pb}-\mathrm{Pb}$ collisions compared to calculations from YaJEM [8] and JEWEL [9]. The boxes at $R_{A A}=1$ represent the systematic uncertainty on $<T_{A A}>$.

\section{Azimuthal Anisotropy of charged jet production}

In non-central $\mathrm{Pb}-\mathrm{Pb}$ collisions, the initial overlap region of the colliding nuclei projected onto the plane perpendicular to the beam direction has an approximately elliptic shape. Jets emitted along the minor axis of the ellipse (defined as the in-plane direction) on average traverse less medium and are therefore expected to lose less energy - than jets that are emitted along the major axis of the ellipse (the out-of-plane direction). The dependence of jet production on the angle relative to the second-harmonic symmetry plane $\Psi_{2}$ (the symmetry plane angles $\Psi_{n}$ define the orientations of the symmetry axes of the initial nucleon distribution of the collision) can be used to probe the path-length dependence of jet energy loss. This dependence is quantified by the parameter $v_{2}^{\text {ch jet }}$, the coefficient of the second term in a Fourier expansion of the azimuthal distribution of jets relative to symmetry planes $\Psi_{n}$

$$
\frac{\mathrm{d} N}{\mathrm{~d} \varphi_{\text {jet }}-\Psi_{n}} \propto 1+\sum_{n=1}^{\infty} 2 v_{n}^{\mathrm{jet}} \cos \left[n\left(\varphi_{\mathrm{jet}}-\Psi_{n}\right)\right]
$$

where $\varphi_{\text {jet }}$ denotes the azimuthal angle of the jet.

Experimentally, the event plane angles $\Psi_{E P, 2}$ and $\Psi_{E P, 3}$ in this study [7], are reconstructed using the VZERO scintillators located at $2.8<\eta<5.1$ and $3.7<\eta<1.7$. Measuring the event plane angles at forward rapidities removes short-range correlations between event planes and tracks. We measure $v_{2}^{\text {ch jet }}$ of charged jets relative to the second harmonic event plane angle. To avoid a bias of the measurement due to the correlation of the background with the event plane, the azimuthal anisotropy of the underlying event is modeled using the flow harmonics $v_{2}$ and $v_{3}$

$$
\rho_{c h}(\varphi)=\rho_{0}\left(1+2\left\{v_{2} \cos \left[2 \varphi-\Psi_{E P, 2}\right]+v_{3} \cos \left[3 \varphi-\Psi_{E P, 3}\right]\right\}\right) .
$$

where $\rho_{c h}$ is the azimuthal distribution of the summed track $p_{\mathrm{T}}$ for tracks with $0.15<p_{\mathrm{T}}<5$ $\mathrm{GeV} / c$ and $|\eta|<0.9$. The parameters $\rho_{0}$ and $v_{n}$ are determined event-by-event from a fit to the data, as illustrated in Fig. 2. 
The corrected transverse jet momentum $p_{\mathrm{T}}^{\text {ch jet }}$ is obtained by subtracting the local underlying event energy $\rho_{\text {ch local }}$, multiplied by the jet area $A$, from the raw jet momentum, $p_{\mathrm{T}}^{\text {ch jet }}=p_{\mathrm{T}, \text { ch jet }}^{\text {raw }}-\rho_{\text {ch local }} A$, where $\rho_{\text {ch local }}$ is obtained from integration of $\rho_{c h}(\varphi)$ over $\varphi_{\text {jet }} \pm R$,

$$
\rho_{\text {ch local }}=\frac{\left\langle\rho_{\text {ch }}\right\rangle}{2 R \rho_{0}} \int_{\varphi-R}^{\varphi+R} \rho_{c h}(\varphi) \mathrm{d} \varphi .
$$
yields

The coefficient $v_{2}^{\text {ch jet }}$ is calculated from the difference between the unfolded $p_{\mathrm{T}}$-differential jet

$$
v_{2}^{\text {ch jet }}\left(p_{\mathrm{T}}^{\text {ch jet }}\right)=\frac{\pi}{4} \frac{1}{\mathcal{R}_{2}} \frac{N_{\text {in }}\left(p_{\mathrm{T}}^{\text {ch jet }}\right)-N_{\text {out }}\left(p_{\mathrm{T}}^{\text {ch jet }}\right)}{N_{\text {in }}\left(p_{\mathrm{T}}^{\text {ch jet }}\right)+N_{\text {out }}\left(p_{\mathrm{T}}^{\text {ch jet }}\right)} .
$$

The factor $\mathcal{R}_{2}$ corrects $v_{2}^{\text {ch jet }}$ for the resolution of the event plane measurement. To correct the jet spectra for instrumental resolution and region-to-region fluctuations of the underlying event, $N_{\text {in }}$ and $N_{\text {out }}$ are unfolded separately, using independent descriptions of the background energy-density fluctuations.

In Fig. 3 [7], $v_{2}^{\text {ch jet }}$ is presented for central (0-5\%) and semi-central (30-50\%) collisions. Significant positive $v_{2}^{\text {ch jet }}$ is found in semi-central collisions, indicating path-length dependent in-medium parton energy loss. In central collisions, the data hint at a positive $v_{2}^{\text {ch jet }}$, but the larger relative contributions of background to the measured jet energy leads to larger systematic uncertainties and the measured values are found to be compatible with the hypothesis $v_{2}^{\text {ch jet }}=0$. The data are compared to $v_{2}^{\text {ch jet }}$ calculations from the JEWEL [9] jet quenching MC. In semi-central collisions, good agreement with the model is found. JEWEL however currently does not include fluctuations in the participant distribution of nuclei in the nucleus, which may lead to the apparent underestimation of $v_{2}^{\text {ch jet }}$ in central collisions.

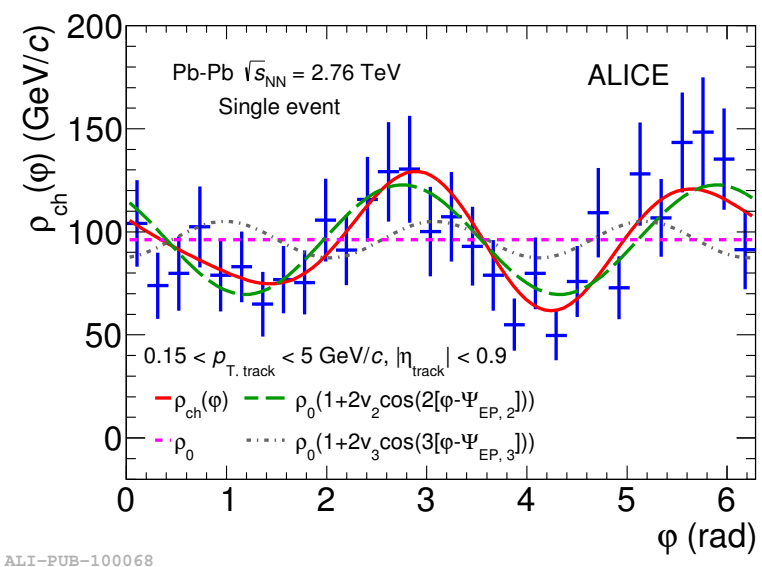

Figure 2. Transverse momentum density of charged tracks as a function of azimuthal angle for a single event from the most central 0-5\% event class [7]. The red curve is the fit of Eq. 1 to the data distribution (blue), the green and gray curves, obtained from the fit of Eq. 1 as well, show the independent contributions of $v_{2}$ and $v_{3}$ to $\rho_{c h}(\varphi)$. The dashed magenta line is the normalization constant $\rho_{0}$. 

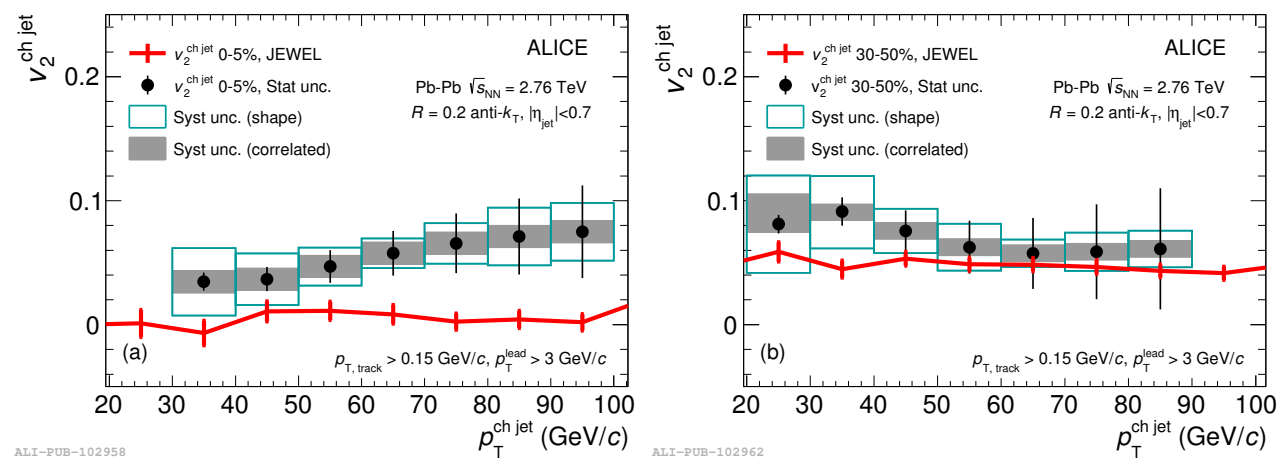

Figure 3. Second-order harmonic coefficient $v_{2}^{\text {ch jet }}$ as a function of $p_{\mathrm{T}}^{\text {ch jet }}$ for $0-5 \%$ (left) and $30-50 \%$ (right) collision centrality [7]. The error bars on the points represent statistical uncertainties, the open and shaded boxes indicate the shape and correlated uncertainties. The data are compared to calculations from the JEWEL MC (red line).

\section{Jet Shapes}

To characterize the intra-jet momentum flow of quenched jets, we focus on two jet shape observables that probe complementary aspects of the jet fragmentation, namely the first radial moment $g$ and the momentum dispersion $p_{\mathrm{T}} D$. The radial moment $g$ is defined as:

$$
g=\sum_{i \in j e t} \frac{p_{\mathrm{T}}^{i}}{p_{\mathrm{T}, \mathrm{jet}}}\left|\Delta R_{i, j e t}\right|
$$

where $p_{\mathrm{T}}^{i}$ stands for the momentum of the constituent $i$ and $\Delta R_{i, j e t}$ is the distance in $\eta, \phi$ space between constituent $i$ and the jet axis. This observable measures the radial energy profile of the jet.

The momentum dispersion $p_{\mathrm{T}} D$ is defined as:

$$
p_{\mathrm{T}} D=\frac{\sqrt{\sum_{i \in j e t} p_{T, i}^{2}}}{\sqrt{\sum_{i \in j e t} p_{T, i}}}
$$

This shape measures the second moment of the constituent $p_{\mathrm{T}}$ distribution in the jet and is sensitive to a possible hardening/softening of the fragmentation of quenched jets.

The shapes are measured in central (0-10\%) collisions for charged jets with a resolution parameter $R=0.2$. The underlying event background is subtracted using the area-derivatives method [10], which numerically determines the susceptibility of a given shape to the background density and extrapolates to zero background. As a check of the validity of the method and to quantify the systematic uncertainty related to the background subtraction, we also use the constituent subtraction [11] algorithm which subtracts background locally and particle-by-particle. Residual effects of background fluctuations and detector effects are corrected by 2-Dimensional Bayesian unfolding as implemented in the RooUnfold [12] package.

The fully corrected jet shapes are shown in Fig. 4. We compare the data to simulations from PYTHIA Perugia 11 [13] in the same jet $p_{\mathrm{T}}$ range of $40-60 \mathrm{GeV} / c$. This PYTHIA tune gives a reasonable description of the jet shapes measured in pp collisions at $\sqrt{s}=7 \mathrm{TeV}$ [14]. The radial moment (left panel) is shifted to lower values in data compared to PYTHIA. The $p_{\mathrm{T}} D$ (right panel) 
is shifted to higher values in data compared to PYTHIA. Our results indicate that the jet cores in $\mathrm{Pb}-\mathrm{Pb}$ are more collimated and harder than the jet cores in PYTHIA at the same energy. Due to jet quenching, when we compare jet shapes in $\mathrm{Pb}-\mathrm{Pb}$ and $\mathrm{pp}$ at the same measured energy, we might bias towards higher initial parton energy in $\mathrm{Pb}-\mathrm{Pb}$ if a significant fraction of the radiated energy is outside the jet cone. Then the question is how the energy was lost and how the radiation pattern of the jet was modified. JEWEL [9] medium-modified jets are narrower and are harder than vacuum jets at the same reconstructed energy, in qualitative agreement with the $\mathrm{Pb}-\mathrm{Pb}$ data as seen in Fig. 4. The underlying physics mechanism in JEWEL leads to a collimation of the jet, where soft modes are transported to large angles relative to the jet axis.
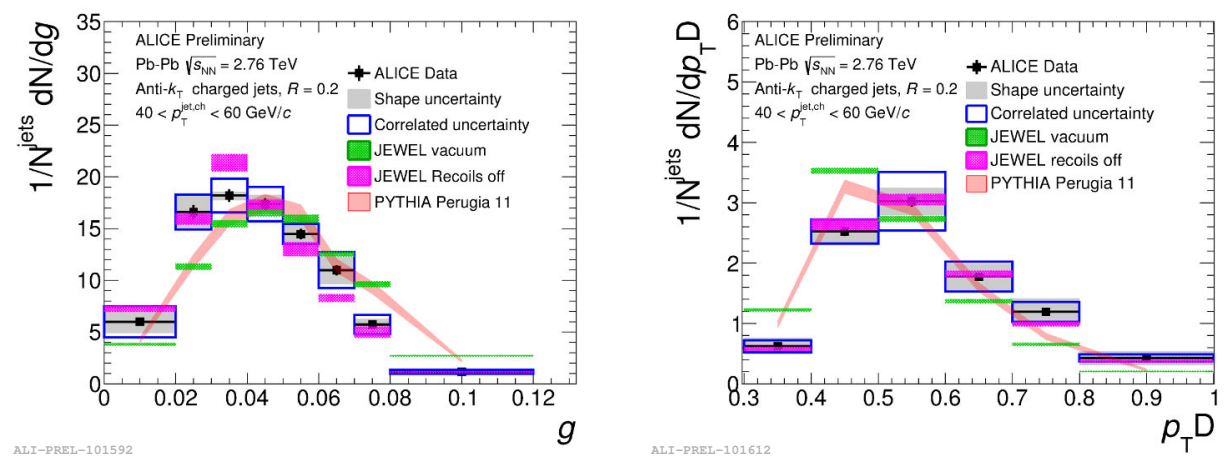

Figure 4. Jet shape distributions for charged jets with $R=0.2$ in $\mathrm{Pb}-\mathrm{Pb}$ collisions compared to PYTHIA simulations [13] and to the JEWEL model [9].

\section{Summary}

Results on charged and full jet production in $\mathrm{Pb}-\mathrm{Pb}$ collisions at $\sqrt{s_{N N}}=2.76 \mathrm{TeV}$ were reported. The nuclear modification factor of jets reconstructed with a resolution parameter $R=0.2$ shows a strong, centrality dependent suppression with respect to pp collisions. The observed value of the jet nuclear modification factor is similar to the $R_{A A}$ of charged hadrons, which indicates that a significant fraction of the lost parton energy is not recovered inside the jet cone. This conjecture is confirmed by the measurement of jet shapes. The distributions of $g$ and $p_{\mathrm{T}} D$ measured in $\mathrm{Pb}-\mathrm{Pb}$ collisions are different from the PYTHIA pp reference, indicating a collimation and hardening of the jet core in $\mathrm{Pb}-\mathrm{Pb}$ relative to pp collisions. The effects are qualitatively reproduced by the JEWEL jet quenching model. The azimuthal anisotropy of charged jet production relative to the event plane indicates a significant path-length dependence of the in-medium parton energy loss in semi-peripheral events. In central events, there are indications for a non-zero $v_{2}^{\text {ch jet }}$. The measurements in peripheral events are well described by the JEWEL MC incorporating collisional and radiative energy loss.

\section{References}

[1] R. Baier, Y. Dokshitzer, S. Peigné, D. Schiff, Phys. Lett. B 345, 277 (1995)

[2] F. Dominguez, C. Marquet, A.H. Mueller, B. Wu, B.-W. Xiao, Nucl. Phys. A 811, 197 (2008)

[3] C. Marquet, T. Renk, Phys. Lett. B 685, 279 (2010) 
[4] M. Cacciari, G. P. Salam and G. Soyez, EPJ 72, 1896 (2012)

[5] J. Adam et al. [ALICE Collaboration], Phys. Lett. B 746, 1 (2015)

[6] B. Abelev et al. [ALICE Collaboration], Phys. Rev. C 88, 044909 (2013)

[7] J. Adam et al. [ALICE Collaboration], Phys. Lett. B 753, 511 (2016)

[8] T. Renk, Phys. Rev. C 88, 014905 (2013)

[9] K. C. Zapp, F. Krauss, U. A.Wiedemann, JHEP 1303, 080 (2013)

[10] G. Soyez, G. P. Salam, J. Kim, S. Dutta and M. Cacciari, Phys. Rev. Lett. 110, 16 (2013)

[11] P. Berta, M. Spousta, D. W. Miller and R. Leitner, JHEP 1406, 092 (2014)

[12] http://hepunx.rl.ac.uk/adye/software/unfold/RooUnfold.html

[13] P. Z. Skands, Phys. Rev. D 82, 074018 (2010)

[14] L. Cunqueiro [ALICE Collaboration], Nucl. Phys. A 956, 593 (2016)

[15] B. Abelev et al. [ALICE Collaboration], Phys. Lett. B 72052 (2013) 